\title{
Health Education, Recent and Future Trends
}

\author{
André Giordan
}

\begin{abstract}
Laboratoire de Didactique et Epistémologie des Sciences, Université de Genève, FPSE - 9 route de Drize,
\end{abstract} CH 1227 Carouge, Genève, Switzerland

During the past, Health Education has been taking place in a variety of ways: prevention, monitoring and control of potentially epidemic diseases. New trends have been arising (such as 'health corners', interdisciplinary activities, exhibit, 'mini-university' for children, etc.). But it is important to discuss what 'Health Education' means, and define 'health' and rethink educational strategies. Several evaluations have highlighted the limited impact that communication activities, or one-off awareness campaigns, may have.

Key words: health education - conception - allosteric-learning model

During the past 30 years, Health Education has been taking place in a variety of ways. There have been awareness campaigns towards the prevention, monitoring and control of potentially epidemic diseases. Some have targeted specialists, by sharing information on the progress and problems surrounding the control of epidemics. Others were addressed to the public, for example prevention campaigns on tuberculosis, leprosy, polio, Chagas disease etc., following in the wake of 19th century hygiene campaigns. The use of radio, cinema, television and other communication media were called for in such cases.

During the 70's, parental worries about drug use led to more specific interventions, which were meant to warn children of its dangers. In many cases, one-off classroom interventions were supposed to 'vaccinate' students, in a medical, preventive and cognitive approach. But doubts regarding the efficiency of such interventions stimulated a reflection process in the 80 's, and the emergence of a more systematic Health Education, with a global and positive approach to health, covering many topics such as prevention, hygiene, safety, first aid, and so on. Further, biology lessons were broadened to include the dimension of health, mainly in primary and in early secondary school. The most frequently developed topics are dietary, bodily and dental hygiene, the prevention of sexually transmitted diseases (STD), especially since the advent of Aids, sexual education, with a focus on contraception, and drugs.

Fax: 41-22-705.9828. E-mail: giordan@uni2a.unige.ch Received 7 August 2000

Accepted 4 September 2000

\section{RECENT TRENDS}

Since new trends have been arising, for example, schools may have 'health corners', staffed by a nurse, various discussion topics are proposed, ranging from eating disorders to tobacco-related cancers or vaccinations. Also, in the realm of 'health club' activities, adolescents may undertake studies on drugs (including alcohol, tobacco, and medicinal drug abuse). Further, interdisciplinary activities (linking biology, physical education, etc.) can promote the understanding of bodily functions (physiology, diet, etc.), leading to the creation of a journal, or an exhibit.

Our team has introduced a number of innovations through special activities, exhibits, and a 'mini-university' for children (De Vechi \& Giordan 1989). We have addressed personal topics, such as an 'introduction to taste', or 'dealing with stress'. This may include practical relaxation exercises, the study of sleeping rhythms and household accidents when working with children, or the issue of road accidents involving bikes and mopeds with adolescents.

We try to link health and the society with topics such as violence, sanitary problems, or controversies surrounding the food business. In Europe, we are currently having problems with certain production methods (e.g. mad cow disease, listeria). In vocational schools we address the consequences of specific technologies (asbestos, paints, etc.). We relate health to the environment by discussing environment-related cancers (heavy metals, dioxin, non-ionising pollution, and so on). Cell phones, which are widespread among teenagers, and the multiplication of transmission masts in cities are an excellent starting point. Further, we participate in health maintenance programs for people with chronic illnesses, such as diabetes, asthma or Parkinson's disease. 
But we must now delve more deeply into several of these issues. First, we must discuss what 'Health Education' means, and define 'health'. Second, we must rethink our educational strategies. Several evaluations have highlighted the limited impact that communication activities, or oneoff awareness campaigns, may have.

\section{SINGULAR ASPECTS OF HEALTH EDUCATION}

Health Education particularities - Health Education has certain particularities, which distinguish it both from health care in itself and from curricula in general (Fig. 1). For health professionals (doctors, nurses, etc.), it is unusual to focus on health in a positive way, and to interact with healthy discussion partners. Educational relationships are different from the caring relationships for which they are trained, and prescriptions must be replaced by dialog. In comparison with other school topics, health knowledge is quite different, as it is not commonplace for students to be taught something about their person, their intimacy, or about social taboos like sexuality or death. It requires increasingly interdisciplinary approaches, related to environmental education and education towards citizenship.

Concept of 'health' - Regarding the concept of 'health': what can we say? This complex concept is defined in several ways. The WHO's model of health integrates several reference categories. Knowledge on health is not only medical. The WHO's definition has the advantage of including psychosocial dimensions, and not only biological ones. It also embodies an utopian view, which must be put into perspective, considered a guideline, a compass pointing to North. Today, we must integrate environmental dimensions as well. The growing number of cancers makes us suspect environmental parameters. Likewise, a number of developing diseases are linked to social conditions of life, or to the new production and consumption methods, as mentioned earlier.

We must also discuss the principle of precaution. How should it be expressed, depending on the situation, or population? It must be situated are we talking of 'measured risks' or 'calculated risks', 'reasonable risks', or 'controlled risks'? By merely institutionalizing prudence, an impression of fake security is provided, and all experimentation and potential change are forbidden. But are not some of our current farming practices, involving herbicides and pesticides, more risky than certain genetic modified organisms (GMOs)?

By systematically collecting health definitions from varied sources, we find that each human group, at each moment in its history, elaborates its own version of health, which fits in with a given conception of life, projects, constraints and possibilities (Giordan 1998). We should not be surprised. Except in situations when it is under immediate threat, health remains a relative value, whose main benefit is to allow us to enjoy what life offers in the best possible way. Each social group, each

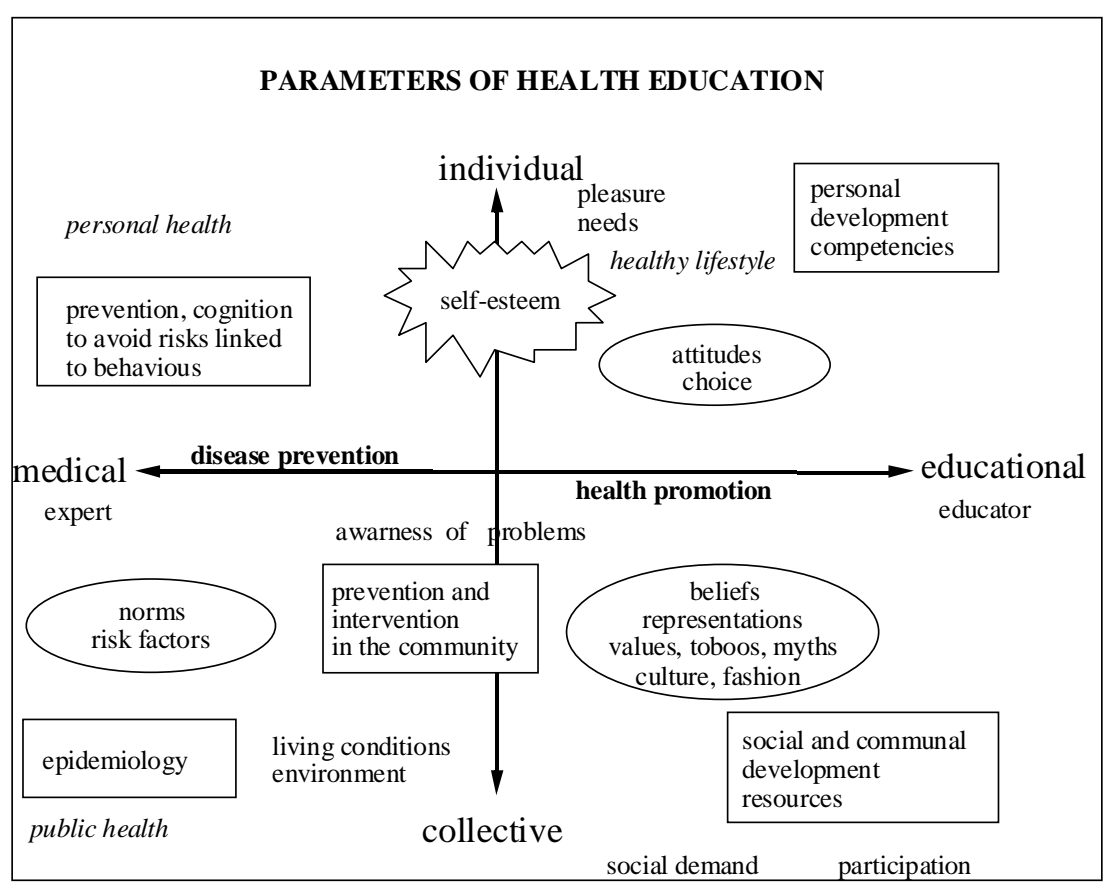

Fig. 1: parameters of health education; adapted from Adler D, LDES internal document, 1998 
human being, has its own needs, both in reality and in dreams. Different strategies are implemented to satisfy them. Individual conceptions of health are operational with respect to individual projects. Knowledge regarding health is therefore not purely cognitive. Above all, attitudes and relational competencies are involved, including the ability to take concrete initiatives and to manage situations, i.e. to know how to act.

By making such relational aspects explicit, we may challenge certain school or family habits. Health education cannot avoid such issues. It must also allow values to be clarified, and other values to be promoted. Certain social activities may not necessarily be good for our diet! Health Education stands out because of its values. Therefore, it is important to implement conditions which recognize this specificity. Currently, there is a growing interest in social development, and in communities, which provides the opportunity for schools and neighborhood to discuss living conditions, and mobilize resources, often with outside collaborators such as associations, social workers, or artists. To avoid pathological references, we should maybe even get rid of the word 'health' altogether, and talk about Education for Life, or for 'Joie de Vivre' - the 'Enjoyment of Life'!

\section{WHICH STRATEGIES?}

Education for prevention is an urgent necessity in hospitals, and in health care institutions, but not only. It is a challenge for our changing society. Yet, the evaluations of previous campaigns highlight the great naiveté of many attempts. So far, the 'pedagogic' model has mainly been frontal, as if 'showing' and 'telling' were enough for the message to 'get through'. Tobacco, for example, is a well-known risk, which accounts for at least 4 million deaths each year on our planet. But this problem brings us back to an essential issue: the gap between 'saying' something and 'doing' it.

In Health Education, knowledge is not enough; we must be able to mobilize our knowledge. It is not obvious to change our behavior. Some still believe that the problem can be solved with publicity, or communication campaigns. Others consider it a question of simple transfer - yet we can easily observe the inefficiency of prevention campaigns targeted towards young people. Obstacles are everywhere: emotional, cognitive and metacognitive ones.

Only multiple and systemic strategies are efficient (Giordan 1995). In Tobacco, only the combination of motors emerging from biology - nicotine desensitizing, psychology - self-image, sociology role models like artists and fashion models, anthropology - behavior of self with others, life habits, and practical ways - new pleasure can have a little chance of being successful. Even 'active' or 'constructivist' methods are quite limited, in particular with health staff (Fig. 2). The conceptions of a targeted public, i.e. their habits, the

Different models on learning
Empirical pedagogy
- a simple recording mechanism
- carried out by a "virgin" and always a vailable brain
- acquisition of knowledge is the direct result of transmission
- In education: the routine and supposedly "logical" presentation of data, now illustrated
- In the museum: the usual exhibition of objects or documents accompanied by notices
a merely linear, frontal relation between a broadcaster (teacher, journalist, museologist
and a receiver (pupil or general public), who memorizes successive messages
Behaviorist education
- training elevated to the rank of principle
- situations accompanied by a questioning which can find immediate answers
learning is favoured by "rewards" (positive reinforcement) or "punishments" (negative
reinforcement) through such conditioning, the individual eventually adopts the right
behaviour
- constructivist education
- advocates their free expression, their creativity and their knowledge of how to be
- propounds independent discovery or the importance of feeling one's way in the learning act
representations

Fig. 2: the characteristics of different models on learning 
things they take for granted, or their ways of reasoning, are all factors which limit the understanding or execution of a message. Examples can be found on children draws, asked for studies on the obstacles under different themes (Figs 3-5). Their draws on how are their mouth and teeth (Fig. 3) depict conceptions that ignore that teeths are separated one from another before the age of 6 year old, when children start to lost their first teeths. Draws of microbes show monster conceptions (Fig. 4) and influences actions on infectious diseases prevention (Fig. 4). Even adult conceptions are often impregnated with misconceptions and errors, as for example, conceptions on digestion (Fig. 5). In Fig. 5a, liver and pancreas precede and are linked to the stomach, and the kidneys are linked to the large intestine. In Fig. 5b the liver is also linked to the stomach and the large precede the small intestines. Adult conceptions on disease depict three different categories (Fig. 6): $93 \%$ direct/causal, $7 \%$ mixed. No systemic conceptions were detected.

Implementing Health Education comes up against our habitual ways of functioning: students must be strongly motivate before (Giordan 1994). They elaborate knowledge from their own lives. They learn through their mental conception, depending on each person's social and biological experience, and individual disposition. The adoption of 'healthy' practices requires many investigations (group discussions, inquiries, confrontations with different information, etc.) which increase awareness about motivations, highlight the diversity of aspects to consider, and anticipate the choice of a certain lifestyle. For prevention, both the deconstruction of certain ways of thinking (or working), and the construction of others are needed. Both the de-connection of one thought, and an alternative neuronal reconnection (Giordan et al. 1994, Giordan \& Girault 1996).

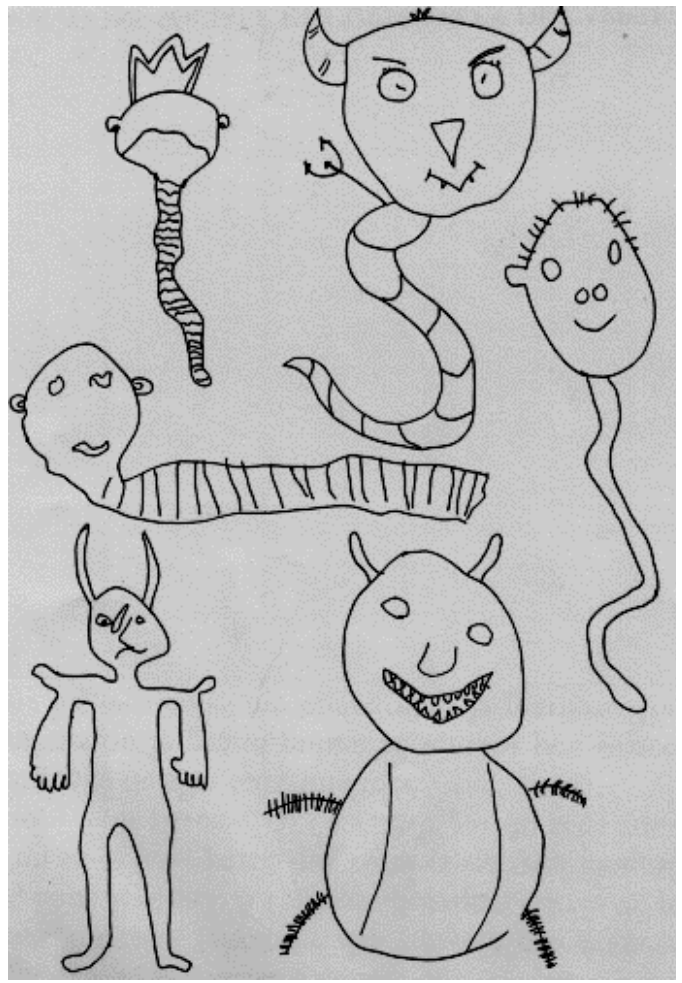

Fig. 4: conception of microbes: draws of 6 year old children

Nothing is direct, nor automatic. Only learners, be they professional or not, can learn. But this will happen only by interacting with a complex, systemic, and often paradoxical, didactic environment (Fig. 7), which encourages both thought transformation... and mobilization. To implement such a heuristic perspective, we need to develop a real health education research in the same time of biology research. a
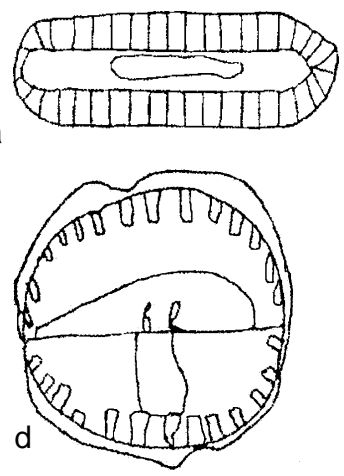
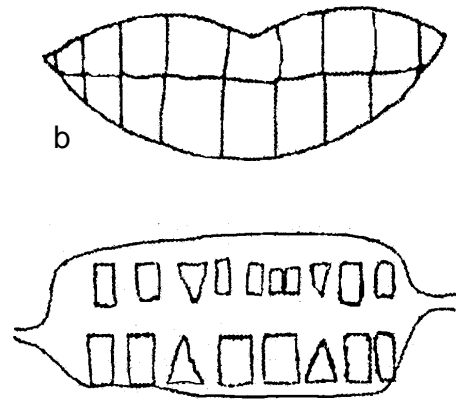

e
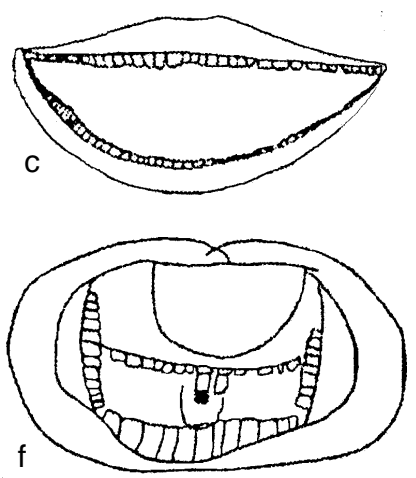

Fig. 3: children draws of their mouth and teeth. Draws at a, b and c were obtained from 4-5 year-old children. Draws d, e, and $\mathrm{f}$ were obtained from 6-7 year old children. 

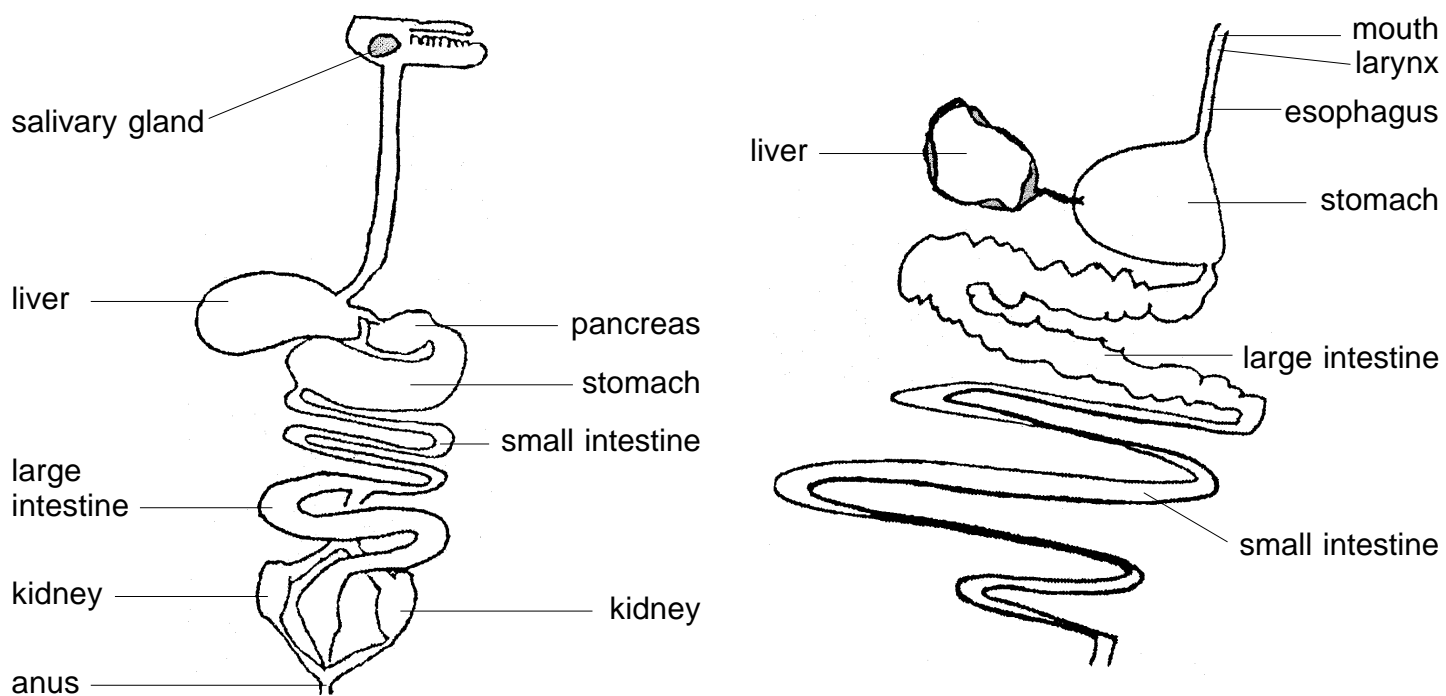

Fig. 5: conception on digestion: draws made by adults. Original words were translated and substituted by typewritten words.

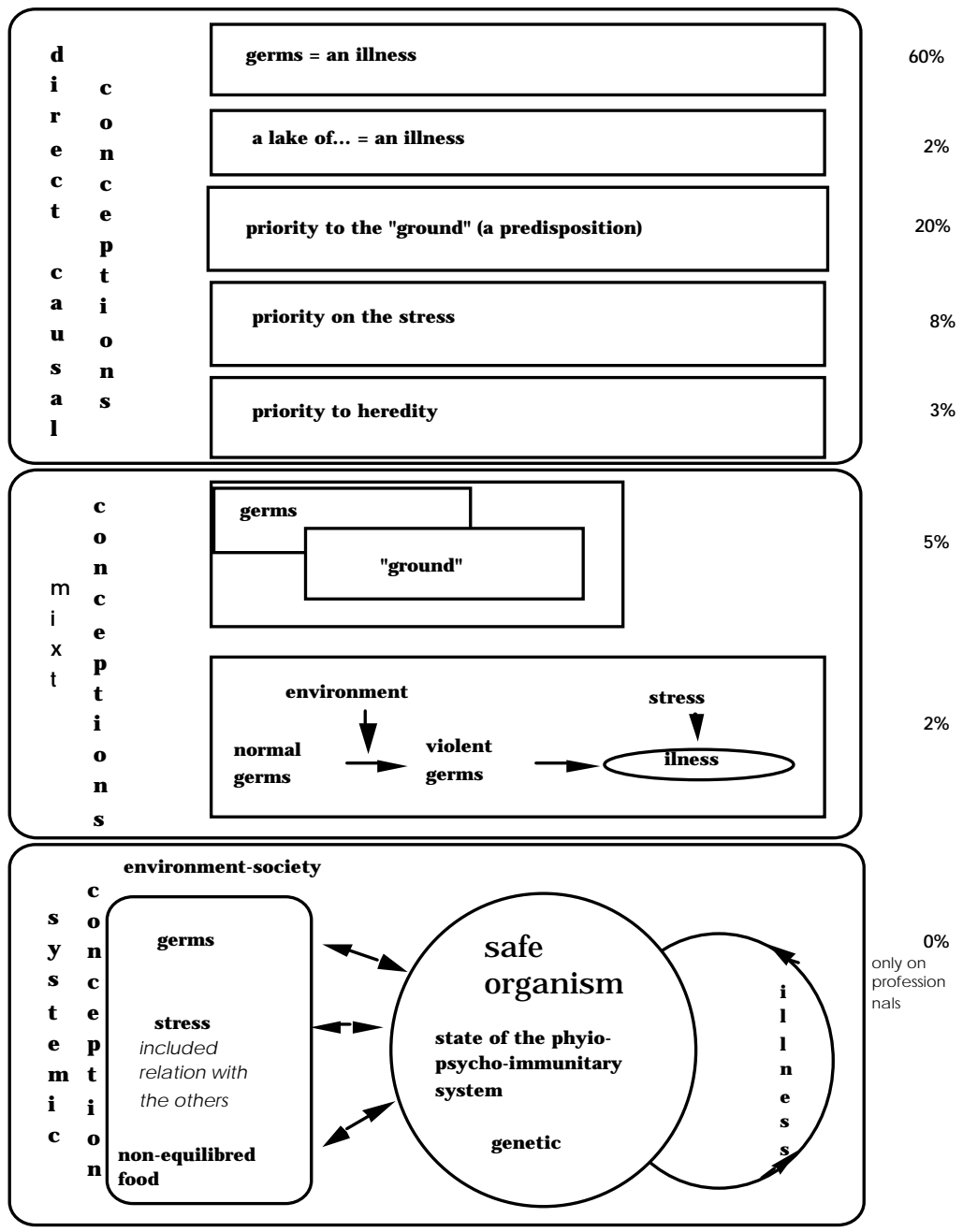

Fig. 6: frequency of individuals in each category of the different conceptions of diseases 


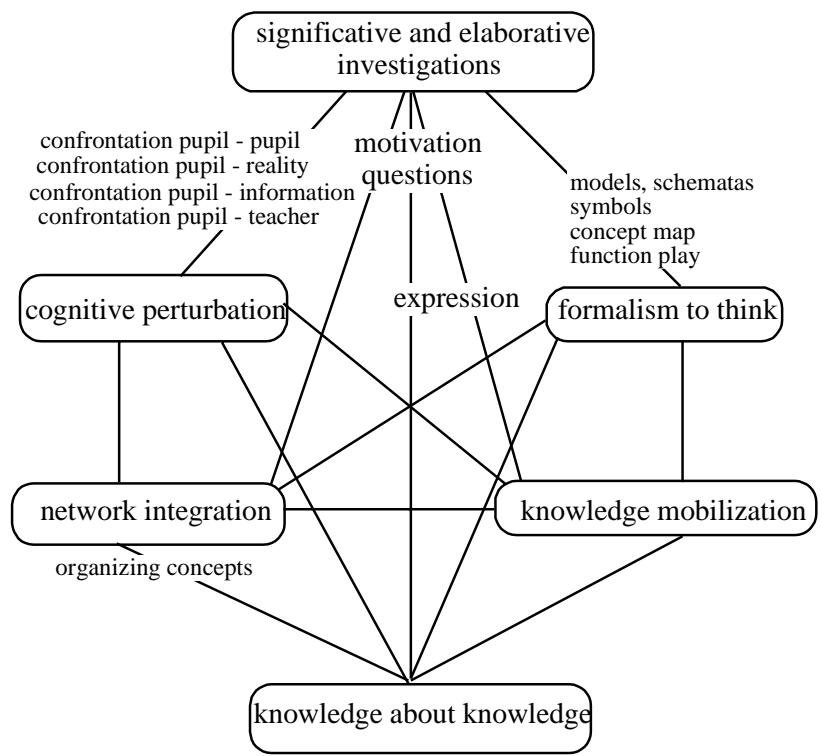

Fig. 7: parameters networks of didactical environment for learning

\section{REFERENCES}

De Vecchi G, Giordan A 1989. L'Enseignement Scientifique: Comment Faire pour que "ça Marche”?, Z' Editions, Nice.

Giordan A 1995. New models for the learning process: beyond constructivism. In Prospects, Vol XXV, 1,

\section{BIE UNESCO.}

Giordan A 1998. Apprendre!, Ed. Belin.

Giordan A, Girault Y 1996. New Learning Models, Z'Editions, Nice.

Giordan A, Girault Y, Clement P 1994. Conceptions et Connaissance, Peter Lang. 